\title{
Modeling the impact of a strong X-class solar flare on the planetary ion composition in Mercury's magnetosphere
}

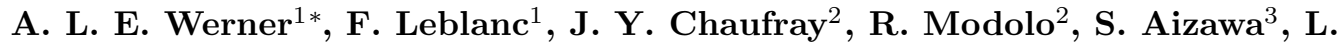 \\ Z. Hadid ${ }^{4}$, C. Baskevitch ${ }^{2}$ \\ ${ }^{1}$ LATMOS/IPSL, Sorbonne Université, UVSQ, CNRS, Paris, France \\ ${ }^{2}$ LATMOS/IPSL, UVSQ Université Paris-Saclay, Sorbonne Université, CNRS, Guyancourt, France \\ ${ }^{3}$ IRAP, Toulouse, France \\ ${ }^{4}$ Laboratoire de Physique des Plasmas (LPP), CNRS, Observatoire de Paris, Sorbonne Université, \\ Université Paris Saclay, Ecole polytechnique, Institut Polytechnique de Paris, 91120 Palaiseau, France
}

\section{Key Points:}

- A strong X-class flare can boost the photoionization frequencies of Mercury's Mg, $\mathrm{O}$ and He exospheres with $40-80 \%$.

- The dayside magnetosphere contains two ion populations for each species which respond to the flare on different time scales.

- Depending on the flare geometry, there may be a time delay between the maximum $\mathrm{Mg}^{+}, \mathrm{O}^{+}$and $\mathrm{He}^{+}$ion densities in the magnetosphere.

${ }^{*}$ Current affiliation: Swedish Institute of Space Physics, Uppsala, Sweden

Corresponding author: Elisabeth Werner, elisabeth.werner@latmos.ipsl.fr 


\begin{abstract}
We model the impact of an extreme solar flare on the $\mathrm{Mg}^{+}, \mathrm{Na}^{+}, \mathrm{O}^{+}$and $\mathrm{He}^{+}$ion density distribution in Mercury's magnetosphere. The Flare Irradiance Spectral Model of the solar irradiance during the X9.3-class flare on 6 September 2017 is used as input to the time-dependent Latmos Ionized Exosphere ion density model. We find that the timeevolution of the planetary ion distribution differs with respect to energy, location and species. There exist two ion energy populations on the dayside that experience different dynamical evolution. The peak ion density in the nightside plasma sheet is delayed by $\sim 7-8$ minutes compared to the dayside. The maximum $\mathrm{Mg}^{+}$density occurs $\sim 4$ minutes before $\mathrm{He}^{+}$and $\mathrm{O}^{+}$in the whole magnetosphere. The time delay between different species does not necessarily occur for solar flares that erupt near the apparent solar limb, where the optical depth is large.
\end{abstract}

\title{
Plain Language Summary
}

A solar flare is a sudden outburst on the Sun which releases radiation and energetic particles. The abrupt radiation enhancement can strongly increase the frequency by which neutral atoms in Mercury's thin atmosphere are ionized. We use a model of the flare radiation spectrum and a new ion density model to study how a strong solar flare impacts the distribution of planetary ions in Mercury's magnetosphere. We select the strongest solar flare of solar cycle 24, which occurred on 6 September 2017. We find that the time-evolution of the ion density varies depending on the planetary ion species, the location inside the magnetosphere, the ion energy and the location of the flare on the Sun with respect to Mercury. The maximum $\mathrm{Mg}^{+}$density occurs $\sim 4$ minutes before $\mathrm{He}^{+}$and $\mathrm{O}^{+}$in the whole magnetosphere. This only happens for solar flares which erupt near the center of the solar disk as seen from Mercury. There are two ion populations with different energies on the dayside, and a single ion population on the nightside. For all species, the peak ion density in Mercury's shadow occurs $\sim 7-8$ minutes after the corresponding peak on the dayside.

\section{Introduction}

Mercury has a tenuous, collision-less atmosphere (i.e. a surface-bounded exosphere) that consists of $\mathrm{H}, \mathrm{He}, \mathrm{Na}, \mathrm{K}, \mathrm{Mg}, \mathrm{Ca}, \mathrm{Mn}, \mathrm{Fe}$ and $\mathrm{Al}$ (Broadfoot et al., 1974; Potter \& Morgan, 1985, 1986; Bida et al., 2000; McClintock et al., 2008; Bida \& Killen, 2017; Vervack et al., 2016). The exosphere is maintained over time by different source and loss mechanisms. Mercury's exosphere is mainly sourced from the surface regolith, diffusion of gases from Mercury's interior and surface bombardment by solar wind ions (Killen et al., 2007). The species are released from the regolith into the exosphere by a variety of ejection processes, such as thermal desorption, photon-stimulated desorption, solar wind ion sputtering and meteoroid impact vaporization (Leblanc \& Johnson, 2003, 2010; Killen et al., 2007). Neutrals are then lost from the exosphere by thermal (Jeans) escape, acceleration of the atoms by the solar radiation pressure to escape velocity and photoionization.

Mercury has a small magnetosphere that is the result of the interaction between the interplanetary magnetic field (IMF) and the intrinsic dipole magnetic field (Anderson et al., 2011). The magnetospheric ion population mainly consists of solar wind ions, but planetary ions may contribute to as much as $10 \%$ of the total ion pressure (Yagi et al., 2010). The planetary ions that exist in Mercury's magnetosphere are primarily sourced from photoionization of the neutral exosphere. The Fast Imaging Plasma Spectrometer (FIPS; Andrews et al., 2007) onboard the Mercury Surface, Space Environment, Geochemistry, and Ranging (MESSENGER) spacecraft has mapped the distribution of planetary ions in Mercury's magnetosphere. $\mathrm{Na}^{+}$-group (mass-per charge ratio $\mathrm{m} / \mathrm{q}=21$ $30 \mathrm{amu} / \mathrm{e}), \mathrm{O}^{+}$-group $(\mathrm{m} / \mathrm{q}=16-20 \mathrm{amu} / \mathrm{e})$ ions and $\mathrm{He}^{+}$were among the most com- 
monly observed ion species by FIPS inside the magnetosphere (Zurbuchen et al., 2011; Raines et al., 2013). The planetary ions were found to be particularly abundant in the central plasma sheet on the nightside and near the northern cusp on the dayside (Raines et al., 2013).

Both Mercury's exosphere (Burger et al., 2014; Cassidy et al., 2015, 2016; Merkel et al., 2017, 2018) and the planetary ion environment (Raines et al., 2013; Jasinski et al., 2021) have been shown to vary as a function of true anomaly angle (TAA). Groundbased observations of the Na exosphere have shown variations with a timescale on the order of hours (Leblanc et al., 2008, 2009; Mangano et al., 2009, 2013, 2015; Orsini et al., 2018) to minutes (Massetti et al., 2017). Changes in the Na emission distribution have been attributed to variations in the solar wind IMF and solar transient events (Mangano et al., 2013, 2015; Orsini et al., 2018; Milillo et al., 2021). Jasinski et al. (2020) determined that a large meteoroid impact event was responsible behind the FIPS observation of a sudden $\left(<10\right.$ minute) enhancement of the $\mathrm{Na}^{+}$-group ion flux $\left(\sim 10^{4} \mathrm{~cm}^{-2} \mathrm{~s}^{-1}\right.$ at $\sim 5300 \mathrm{~km}$ ). Raines et al. (2018) reported an enhancement of the $\mathrm{He}^{+}$density (up to $0.1 \mathrm{~cm}^{-3}$ ) in the northern cusp following the transit of a CME at Mercury.

There have been a number of intense solar flare events in modern time. Noteworthy examples include the Bastille Day event on 14 July 2000 (Aulanier et al., 2000), the Halloween solar storms in 2003 (Tsurutani et al., 2005) and more recently, a set of strong X-class flares in September 2017 (Yan et al., 2018). On Earth, extreme solar flares can give rise to solar radiation storms, which can have severe biological effects and disrupt satellite operations, and radio blackouts, which affects positioning and satellite navigation (National Oceanic and Atmospheric Administration, 2011). Solar flares have also been shown to enhance X-ray emission at Jupiter (Maurellis et al., 2000), Saturn (Bhardwaj et al., 2005) and disturb Mars's ionosphere (Mendillo et al., 2006; Fallows et al., 2015). To our knowledge, the impact of solar flares on Mercury has not been studied before. Considering Mercury's short heliocentric distance and the unique composition of heavy species in Mercury's exosphere, it is a particularly interesting case to consider.

We have developed a model to simulate the impact of a strong X-class solar flare on the ion density distribution of $\mathrm{Mg}^{+}, \mathrm{Na}^{+}, \mathrm{O}^{+}$and $\mathrm{He}^{+}$in Mercury's magnetosphere. The solar flare event and the model are described in Section 2. We describe the key results in Section 3 and discuss their implications in Section 4. Finally, we summarize our findings in Section 5 .

\section{Model and Method}

\subsection{The X9.3-class Solar Flare on 6 September 2017}

Between 4-10 September 2017 the active region (AR) 12673 on the Sun released a series of solar flares and CMEs that impacted Earth and the planet Mars. Two special issues in the Space Weather journal (Knipp, D., 2018) and the Geophysical Research Letters (Diffenbaugh, N., 2018) review the observations that were made from these events and the impact they had on the two planets.

The strongest solar flare of this period (and solar cycle 24) started at 11:53 Universal Time (UT) on 6 September 2017 and reached peak emission at 12:02 UT. The flare was detected by the Geostationary Operational Environmental Satellites (GOES) and ranked as the $14^{\text {th }}$ most intense solar flare observed since measurements began in 1975 (Berdermann et al., 2018). Solar flares are classified by their maximum energy output, which is estimated from measurements in the wavelength range $\lambda=0.1-0.8 \mathrm{~nm}$ by GOES X-ray sensor (XRS). The 6 September flare had a peak energy output of $9.3 \times 10^{-4} \mathrm{~W} / \mathrm{m}^{2}$ and was therefore classified as an X9.3-class event. The strongest solar flare detected to date occurred on 4 November 2003 and was estimated to X28, which makes it at least three times stronger than the 6 September 2017 flare. 
A flare of similar strength (X8.2) erupted on 10 September 2017 from the same active region and hit the planet Mars. Spacecraft observations of Mars's upper atmosphere after the flare showed signs of heating and expansion of the upper atmosphere (Jain et al., 2018), which caused the exosphere and ion density at a given altitude to increase (Elrod et al., 2018; Thiemann et al., 2018). The photochemical escape of $\mathrm{O}$ was also shown to be enhanced as a result of the flare (Thiemann et al., 2018). The 6 and 10 September 2017 flares likely also affected Mercury but there were no spacecraft in orbit around Mercury that could study its effects.

\subsection{The Flare Irradiance Spectral Model-Version 2}

The Flare Irradiance Spectral Model-Version 2 (FISM2; Chamberlin et al., 2020) is an empirical model of the solar spectral irradiance. The solar spectral irradiance is estimated at a heliocentric distance of $1 \mathrm{AU}$ in the wavelength range 0.05 to $189.95 \mathrm{~nm}$ with a spectral cadence of $0.1 \mathrm{~nm}$. FISM2 uses data from the X-Ray Photometer System (XPS) on the Solar Radiation and Climate Experiment (SORCE) in the wavelength range 0-6 nm, the EUV Variability Experiment (EVE) on Solar Dynamics Observatory (SDO) between 6-105 nm and the Solar Stellar Irradiance Comparison Experiment (SOLSTICE; also on SORCE) between 115-190 nm. The FISM2 output is given in a "daily" and "flare" version. The daily output contains the daily average of the solar spectrum for any given day since 1947 until the present. The flare product consists of a modeled spectrum for every $60 \mathrm{~s}$ of the selected day (from 2003 until the present). The FISM2 solar irradiance spectra are available at http://lasp.colorado.edu/lisird/data/fism.

The FISM2 model relies on a set of proxies to represent the irradiance variability in the full wavelength range $(0-190 \mathrm{~nm})$ caused by the solar cycle, solar rotation and solar flares. The solar spectral irradiance variability due to solar flares is estimated using two separate proxies. Measurements from the GOES/XRS B-channel (0.1-0.8 nm) are used to model the gradual (thermal) phase of the solar flare (Priest, 1981). The timederivative of the GOES/XRS-B measurements are used to represent the impulsive (nonthermal) phase (Neupert, 1968). Only the irradiance variation due to the solar cycle and solar rotation is accounted for in the daily product, while the flare product also accounts for the irradiance variation due to real solar flare events.

We use the FISM2 flare output on 6 September 2017 in order to estimate the timeevolution of the photoionization flux for different species during the specified flare event. The FISM2 model has been used in the past to study the 6 and 10 September $2017 \mathrm{X}$ class flares (Chamberlin et al., 2018). To calculate the $\mathrm{Mg}, \mathrm{Na}$, O and He photoionization frequencies we merge the FISM2 spectra $(0-190 \mathrm{~nm})$ with the solar flux model from Killen et al. (2009) between 190-1300 nm and use the theoretical photoionization cross sections from Verner et al. (1996).

\subsection{The Latmos Ionized Exosphere Model}

The Latmos IoniZed Exosphere (LIZE) model is a test-particle model which describes the 3-D ion density distribution of photo-ions derived from Mercury's exosphere. The model is coupled to a Monte Carlo model of the exosphere (EGM; Leblanc \& Johnson, 2010; Leblanc et al., 2017) and a hybrid model of the magnetosphere (LatHyS; Modolo et al., 2016, 2018). We make a separate LIZE simulation for each ion species $\left(\mathrm{Mg}^{+}\right.$, $\mathrm{O}^{+}$and $\mathrm{He}^{+}$). For the $\mathrm{O}$ and $\mathrm{He}$ exospheres, we used the results of EGM described in Werner et al. (2022), whereas for the Mg exosphere those described in Chaufray et al. (2021a, 2021b). The EGM model of the Na exosphere has been described previously in Leblanc and Johnson (2010) and the He exosphere in Leblanc and Chaufray (2011). We find that the 6 September 2017 flare did not cause the Na surface ejection rate by photostimulated desorption to increase or Mercury's surface temperature to rise (which controls the rate of thermal desorption). Surface ejection by ion sputtering or micro-meteoroid 
vaporization are not affected by the solar radiation conditions. Therefore we make the assumption that the neutral $\mathrm{Mg}, \mathrm{O}$ and He exosphere density does not change significantly during the flare. We use the EGM output at true anomaly angle $T A A=180^{\circ}$ (i.e. at aphelion) for all species. For the simulation of the magnetosphere, we use the same set of solar wind and IMF boundary conditions as "case a" described in Aizawa et al. (2021). The LIZE model has been used previously to determine the average ion density and phasespace density distribution of $\mathrm{Na}^{+}, \mathrm{O}^{+}$and $\mathrm{He}^{+}$inside Mercury's magnetosphere (Werner et al., 2022). The model gives a similar average density and spatial distribution as the $\mathrm{Na}^{+}$-group, $\mathrm{O}^{+}$-group and $\mathrm{He}^{+}$ion density observations made by MESSENGER/FIPS (Raines et al., 2013).

For the purpose of this study, we have implemented the capability to use time-dependent input conditions with the LIZE model. We make repeated test-particle injections in the whole simulation volume with a test-particle weight that depends on the nominal 3-D ion production rate and the time-dependent photoionization frequency calculated with the FISM2 model. We use a 4-D grid $(r, \phi, \theta, E)$ where $r$ is the distance from the planet, $\theta$ is the co-latitude, $\phi$ is the longitude and $E$ is the kinetic energy. The grid is centered on the planet and the simulation volume is bounded between $r=1.0-3.5$ Mercury radii $\left(\mathrm{R}_{M}\right), \theta=0-\pi \mathrm{rad}$ and $\phi=0-2 \pi \mathrm{rad}$. The grid is divided into 65 exponentially distributed cells along $r(\Delta r=5-600 \mathrm{~km}), 40$ cells along $\theta(\Delta \theta=0.08 \mathrm{rad})$ and 60 cells along $\phi(\Delta \phi=0.1 \mathrm{rad})$. The energy range is $E=1-10^{5} \mathrm{eV}$ and the energy resolution is described by the formula $\left(E_{i}-E_{i-1}\right) / E_{i}=0.1$ where $E_{i}$ is the $i$ th energy step. All test-particles inside the simulation are synchronously advanced in space after every time step $(d t=0.01 \mathrm{~s})$. Every $60 \mathrm{~s}$ we inject 50 test-particles with zero initial velocity from random positions within each cell on the grid that has a non-zero ion production rate (as defined in the corresponding EGM simulation). The output consists of "snapshots" of the 3-D ion density distribution. Before triggering the solar flare we initialize the simulation volume with 30 minutes of test-particle injections with weights which correspond to the nominal photoionization frequency (for each species) in order to have a steady state situation of the magnetospheric environment. After this time, the deviation between snapshots taken $60 \mathrm{~s}$ apart is less than $10 \%$.

\section{Results}

\subsection{Time-evolution of the $\mathrm{Mg}, \mathrm{Na}, \mathrm{O}$ and $\mathrm{He}$ photoionization frequency}

Figure $1 a$ shows the integrated solar spectral irradiance during the first 30 minutes of the 6 September 2017 flare event. To make this particular plot we have used the wavelength range $0-190 \mathrm{~nm}$ as opposed to the whole wavelength range $(0-1300 \mathrm{~nm})$, to more clearly show the peaks of the impulsive $(t=3 \mathrm{~min})$ and gradual $(t=6-7 \mathrm{~min})$ phases of the flare. The flare emission that occurs during the impulsive phase is believed to be due to non-thermal acceleration of high speed electrons and protons inside magnetic loops in the solar atmosphere, while the gradual phase is dominated by thermal radiation or bremsstrahlung from the hot gas nested inside the magnetic loops (Dennis $\&$ Schwartz, 1989). Figure $1 b$ shows the solar spectral irradiance at two discrete wavelengths: $\lambda=12 \mathrm{~nm}$ and $\lambda=180 \mathrm{~nm}$. The spectral irradiance at $\lambda=12 \mathrm{~nm}$ is dominated by the gradual phase while the relatively cool, impulsive phase typically dominates at longer wavelengths. Figure 1c shows the time evolution of the photoionization frequency for $\mathrm{He}, \mathrm{O}, \mathrm{Mg}$ and $\mathrm{Na}$ normalized to their values before the start of the flare.

$\mathrm{Na}$ has the highest nominal photoionization frequency of the four species $(5.0 \times$ $10^{-6} \mathrm{~s}^{-1}$ ), but the solar flare has a negligible effect on $\mathrm{Na}$ (see the inset plot in Figure 1c). The Mg photoionization frequency is an order of magnitude smaller compared to $\mathrm{Na}\left(4.5 \times 10^{-7} \mathrm{~s}^{-1}\right)$ but increases with up to $87 \%$ as a result of the flare. The He and $\mathrm{O}$ photoionization frequencies have a similar time-evolution during the flare (see Figure 1c) but have different magnitude (He: $7.5 \times 10^{-8} \mathrm{~s}^{-1}$; O: $3.1 \times 10^{-7} \mathrm{~s}^{-1}$ ). The He den- 

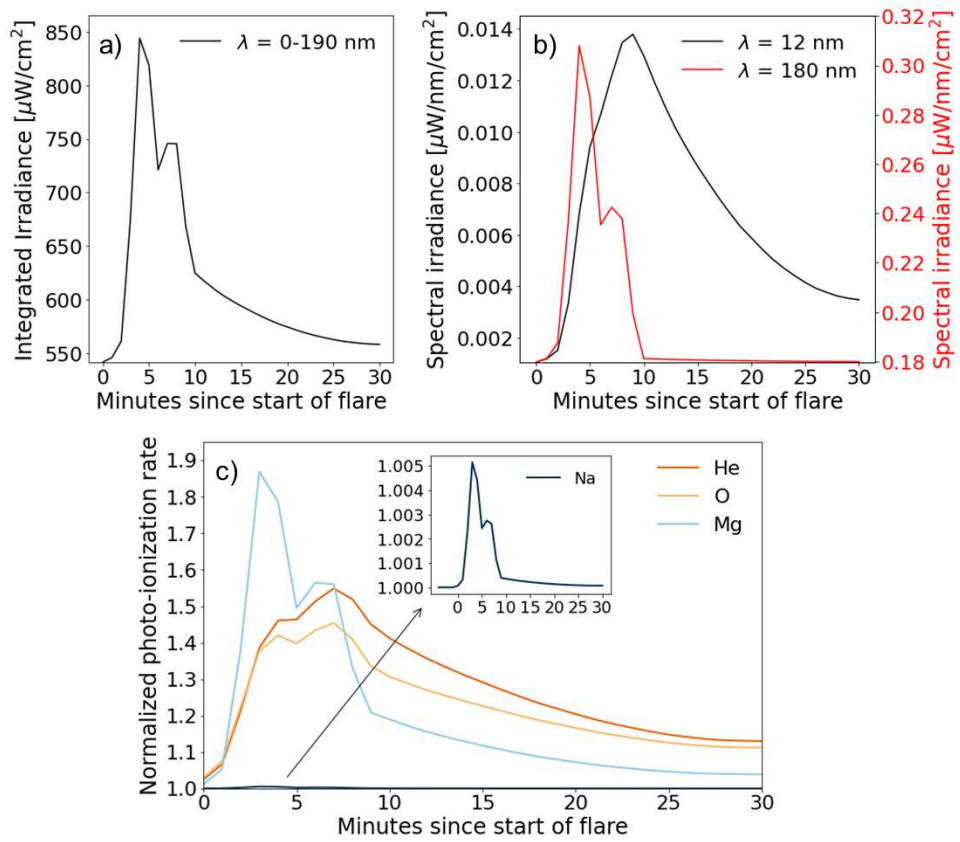

Figure 1. The $(a)$ integrated solar irradiance during the first 30 minutes of the 6 September 2017 flare in the wavelength interval $\lambda=0$ - $190 \mathrm{~nm}$ calculated using the FISM2 model, $(b)$ the spectral solar irradiance at the wavelengths $\lambda=12 \mathrm{~nm}$ and $\lambda=80 \mathrm{~nm}$ and $(c)$ the normalized photoionization frequency for $\mathrm{Na}, \mathrm{He}, \mathrm{O}$ and $\mathrm{Mg}$.

sity from the EGM, which is used as input to the LIZE model, is much higher and have a larger scale height compared to the O density (Werner et al., 2022). The Mg photoionization frequency is highest after 3 minutes, while the maximum He and $\mathrm{O}$ photoionization frequencies occurs 7 minutes after the start of the flare. This implies that the impulsive flare phase is most effective in raising the $\mathrm{Mg}$ photoionization frequency while the gradual phase is more important for He and O. The different time-evolution of the photoionization frequency for each species and their distribution in the exosphere have the potential to create large differences between their ion counterparts in the magnetosphere.

\subsection{Time-evolution of the ion density separated by energy}

\subsubsection{The ion energy spectrum before the flare}

Figure $2 a-c$ show the average $\mathrm{He}^{+}, \mathrm{O}^{+}$and $\mathrm{Mg}^{+}$ion density in the latitude range $\pm 30^{\circ}$ centered on the geometric equatorial plane. We study the evolution of the $\mathrm{He}^{+}$, $\mathrm{O}^{+}$and $\mathrm{Mg}^{+}$ion density as a function of time and energy (Figure 2d-1) inside three different regions in the magnetosphere (black boxes in $2 a-2 c$ ). The energy spectra in Figure $2 \mathrm{~d}-\mathrm{l}$ shows the ion density separated per energy bin and has the unit $\mathrm{cm}^{-3} \cdot d E^{-1}$, where the energy bin width $d E$ is given by $d E=0.1 E_{i}$ and $E_{0}=1 \mathrm{eV}$. The first region (i.e. Region $\mathrm{A}$ ) is located near the surface (Altitude: $0-500 \mathrm{~km}$ ) on the dayside (Local time: 10:30-12:00 h). Region B is located at higher altitudes (Altitude: 100-1100 km) near the dawn terminator (Local time: 05:00-06:30 h), and Region C is located near midnight in the nightside plasma sheet (Altitude: 700-1500 km; Local time: 23:00-01:00 h). Figure $2 d-l$ show the ion energy distributions (energy spectra) for $\mathrm{He}^{+}, \mathrm{O}^{+}$and $\mathrm{Mg}^{+}$ in Region A-C as a function of time. 

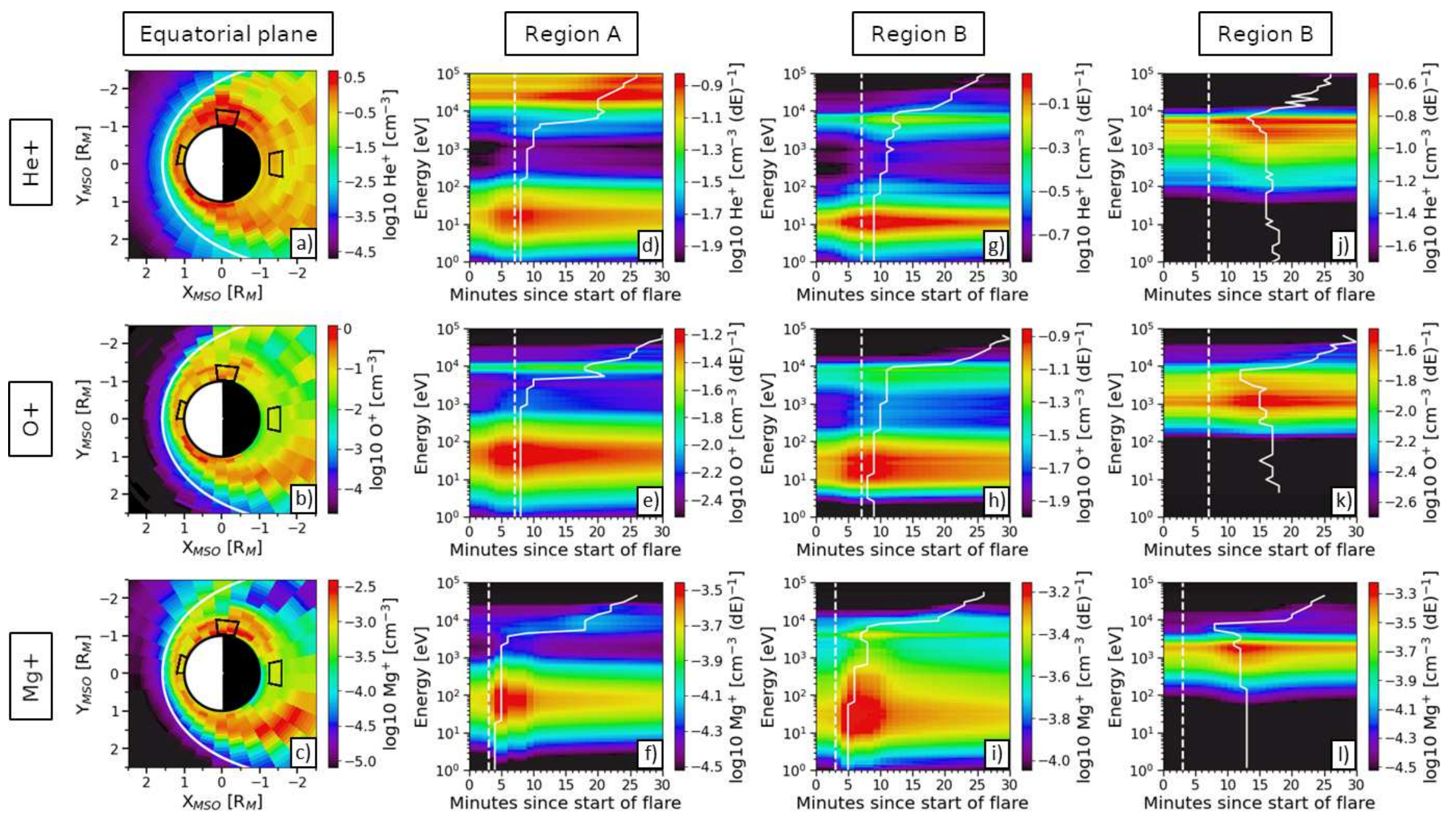

Figure 2. The $(a) \mathrm{He}^{+}(\mathrm{t}=8 \mathrm{~min}),(b) \mathrm{O}^{+}(\mathrm{t}=8 \mathrm{~min})$ and $(c) \mathrm{Mg}^{+}(\mathrm{t}=5 \mathrm{~min})$ ion density in the equatorial plane (average over latitude range $\left.\pm 30^{\circ}\right)$ and $(d-$

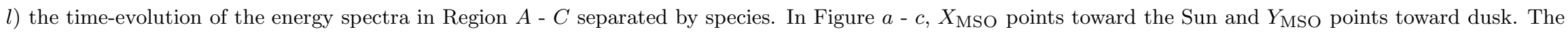
solid white hyperbolas in panels $a-c$ show the location of the magnetopause boundary, which has been calculated and corrected for the solar wind ram pressure of our simulation $\left(P_{\text {ram }}=8 \mathrm{nPa}\right.$ ) following the scheme described in Winslow et al. (2013). The black boxes show the location of Region $A-C$. The dashed white lines in Figure $d-l$ highlights the time when the photoionization frequency for each species reaches its maximum value. The solid white curves identifies the time when the ion density is highest in each energy channel. 
Before the flare $(t=0)$, the energy spectra in Region A-B exhibits two distinctive peaks (Population 1 and 2). Population 1 consists of low-energy ions $(E=0-100$ $\mathrm{eV})$ while Population 2 contains much hotter ions $(E>10 \mathrm{keV})$. The low energy of the ions in Population 1 indicate that they have recently been photo-ionized and were likely created inside or near Region A-B. On the contrary, Population 2 must either contain ions which have been created elsewhere and/or have experienced a different dynamical evolution compared to the ions in Population 1 (see Section 4 for an in-depth discussion). For $\mathrm{He}^{+}$in Region A, Population 1 has a maximum at $E=20 \mathrm{eV}$ and Population 2 at $E=20 \mathrm{keV}$. The energy spectra for $\mathrm{O}^{+}$and $\mathrm{Mg}^{+}$in Region A (see Figure $2 e-2 f$ ) also consists of two ion populations. Population 1 (2) has a mean energy of $E=40 \mathrm{eV}$ $(E=10 \mathrm{keV})$ for $\mathrm{O}^{+}$and $E=80 \mathrm{eV}(E=8 \mathrm{keV})$ for $\mathrm{Mg}^{+}$. The density of the Population 1 and $2 \mathrm{He}^{+}$ions in Region A are quite similar, with Population 1 being just $\sim 40 \%$ more abundant than Population 2. However, for $\mathrm{O}^{+}$and $\mathrm{Mg}^{+}$Population 1 completely dominates the energy spectrum and Population 2 only accounts for $\sim 10 \%$ of the total ion density. The mean energy of the two ion populations are generally lower in Region B: Population 1 (2) has a mean energy of $E=10 \mathrm{eV}(E=5 \mathrm{keV})$ for $\mathrm{He}^{+}, E=30$ $\mathrm{eV}(E=8 \mathrm{keV})$ for $\mathrm{O}^{+}$and $E=30 \mathrm{eV}(E=4 \mathrm{keV})$ for $\mathrm{Mg}^{+}$. Region $\mathrm{C}$ appears to be populated by a single ion population with a relatively high average energy of $E=$ $5 \mathrm{keV}$ for $\mathrm{He}^{+}, E=1 \mathrm{keV}$ for $\mathrm{O}^{+}$and $E=2 \mathrm{keV}$ for $\mathrm{Mg}^{+}$.

\subsubsection{Time-evolution of the ion energy spectrum}

The difference between the dashed line and the solid curves in Figure $2 d-2 l$ illustrates the time delay between the maximum photoionization frequency and the maximum ion density in each energy channel. The time delay for Population 1 in Region A is $\Delta t=1-2$ minutes for all modeled species. The time delay for Population 2 is longer, approximately $\Delta t=14-15$ minutes. Similar values are found in Region B. Inside region $\mathrm{C}$ the maximum ion density occurs at $t=14-15$ minutes for $\mathrm{He}^{+}, \mathrm{O}^{+}$and at $t=11$ minutes for $\mathrm{Mg}^{+}$. If we compare the dashed and the solid curves in Figure $2 j$ - $l$ we find that the time delay is $\Delta t=7-8$ minutes irrespective of the species.

Population 1 typically dominates the total ion density in both Region A and B during the entire simulation for all modeled species. However, the $\mathrm{He}^{+}$Population $1(E=$ $0-100 \mathrm{eV})$ in Region A varies between being twice as dense as Population $2(E>10$ $\mathrm{keV}$ ) at $t=8$ minutes, to only $20 \%$ more abundant compared to Population 2 at $t=$ 22 minutes. In effect, this causes the average $\mathrm{He}^{+}$density to decay more slowly in Region $\mathrm{A}$. The average $\mathrm{He}^{+}$density is elevated by $\sim 25 \%$ compared to the background value for almost 10 minutes shortly after the main peak $(t=8 \mathrm{~min})$. This is not the case for $\mathrm{O}^{+}$and $\mathrm{Mg}^{+}$, that do not possess such a large population of high-energy ions in this region.

\section{Discussion}

The photoionization frequency for different neutral species reach their maximum value at different times during a flare. This depends on the photoionization energy threshold and in particular on the wavelength-dependence of the photoionization cross-section for each species. This may cause the impulsive or the gradual flare phase to be the most effective in raising the overall photoionization frequency. The time-evolution of the $\mathrm{Mg}$ (and $\mathrm{Na}$ ) photoionization frequency exhibit a strong correlation with the impulsive phase of the 6 September 2017 flare (see Figure 1c) while the He and O photoionization frequencies reach their maximum values during the gradual flare phase. This result implies that a spacecraft (which carries a plasma mass spectrometer) in orbit around Mercury during a strong X-class flare event will first detect an increase of the $\mathrm{Mg}^{+}$density followed by $\mathrm{He}^{+}$and $\mathrm{O}^{+}$several minutes later, regardless of where the spacecraft is located inside the magnetosphere. Calculations show that most species that have been observed 
in Mercury's exosphere (Bida et al., 2000; Bida \& Killen, 2017; Broadfoot et al., 1974; McClintock et al., 2008; Potter \& Morgan, 1985, 1986; Vervack et al., 2016) or are expected based on observations of Mercury's surface composition (Evans et al., 2012, 2015; Nittler et al., 2011; Peplowski et al., 2012, 2015) are most affected by the impulsive phase of the 6 September 2017 flare (i.e. H, C, Na, Mg, Al, Si, S, Ar, Ca, Fe). The strength of the impulsive and gradual phase vary on an event-to-event basis. The impulsive flare phase tends to be the dominant phase for small flares, while strong flares like the 6 September 2017 flare often exhibit a relatively strong gradual phase which can last for over an hour (Dennis \& Schwartz, 1989).

At most, there are $2.4 \times 10^{26}\left(\mathrm{He}^{+}: 43 \%\right.$ increase $), 4.5 \times 10^{25}\left(\mathrm{O}^{+}: 38 \%\right.$ increase $)$ $5 \times 10^{23}\left(\mathrm{Mg}^{+}: 49 \%\right.$ increase $)$ additional $\mathrm{He}^{+}, \mathrm{O}^{+}$and $\mathrm{Mg}^{+}$ions being produced respectively in and outside Mercury's magnetosphere. The maximum $\mathrm{He}^{+}, \mathrm{O}^{+}$and $\mathrm{Mg}^{+}$ion production during the flare is equal to barely $0.1 \%$ of the plasma mass density of the $\mathrm{Na}^{+}$ ion population however, and therefore does not cause any significant mass loading of Mercury's magnetosphere.

Analysis of test-particle trajectories for Population 2 ions reveal that they experience a different dynamical evolution compared to Population 1. Population 2 largely consists of ions which have become quasi-trapped in the closed field line region near Mercury's magnetic equator. Figure 3 shows an example $\mathrm{Mg}^{+}$test-particle trajectory from the LIZE model which is typical to Population 2. The $\mathrm{Mg}^{+}$test-particle is ejected in the southern hemisphere and travels toward the dayside equatorial region (see Figure 3a-d and $\mathrm{f}$ ). As the test-particle moves into the dayside hemisphere it approaches the magnetopause (see Figure 3e), and encounters the strong electric field near the magnetosheath (see the red part of the trajectory in Figure 3a-d and g). This causes the ion energy to increase from a few hundred $\mathrm{eV}$ to $>10 \mathrm{keV}$ (see Figure 3h) and the test-particle starts to drift around the planet toward the nightside, where it eventually impacts the planet. The small size of Mercury's magnetosphere prevents the formation of a steady ion drift belt. Low-mass ions like $\mathrm{He}^{+}$can make 1-2 complete orbits before impacting the planet or escaping, while heavier ions like $\mathrm{Mg}^{+}$are typically not able to pass the dayside magnetosphere because of their large gyro radii.

The test-particle trajectory in Figure 3 seems to suggest that the Population 2 ions in Region A does not belong to the Type 0 or Type 1 ion populations described in Glass et al. (2021), but could be part of Type 3. Glass et al. (2021) identified different types of $\mathrm{Na}^{+}$test-particle trajectories which could be responsible for the population of $>1$ $\mathrm{keV} \mathrm{Na}{ }^{+}$ions observed in Mercury's northern magnetospheric cusp by FIPS (Raines et al., 2014). Type 0 ions pass through the magnetosheath before crossing the northern cusp, while Type 1 ions move directly into the northern cusp without passing through or coming near the magnetosheath boundary. Any ion which exceeded a distance of $2 \mathrm{R}_{M}$ from the planet before passing through the cusp was categorized as Type 2, based on the relatively coarse grid resolution of the simulation beyond $2 \mathrm{R}_{M}$. Type 3 consists of $\mathrm{Na}^{+}$ ions which comes close to the magnetopause but do not cross into the magnetosheath before passing through the northern cusp. The $\mathrm{Mg}^{+}$ion in Figure 3 is energized to $>$ $10 \mathrm{keV}$ before its closest approach to the magnetopause (see Figure $3 \mathrm{e}$ and $\mathrm{h}$ ). It is possible that Type 3 ions are rare at high latitudes simply because they are easily (quasi)trapped in the closed field line region near the equator and therefore remain at mid-latitudes.

The magnetopause is located farther away from the surface at the dawn terminator compared to the subsolar point due to solar wind aberration. This implies that the solar wind convective electric field have less influence over the ions in Region B compared to Region A, which leads to overall lower ion energies in this region. Region C is located in Mercury's shadow, where there is no local ion production and ions can only be transported here from elsewhere in the magnetosphere. This explains the lack of a low-energy ion population in Region $\mathrm{C}$ and the time delay between the peak ion density in Region 

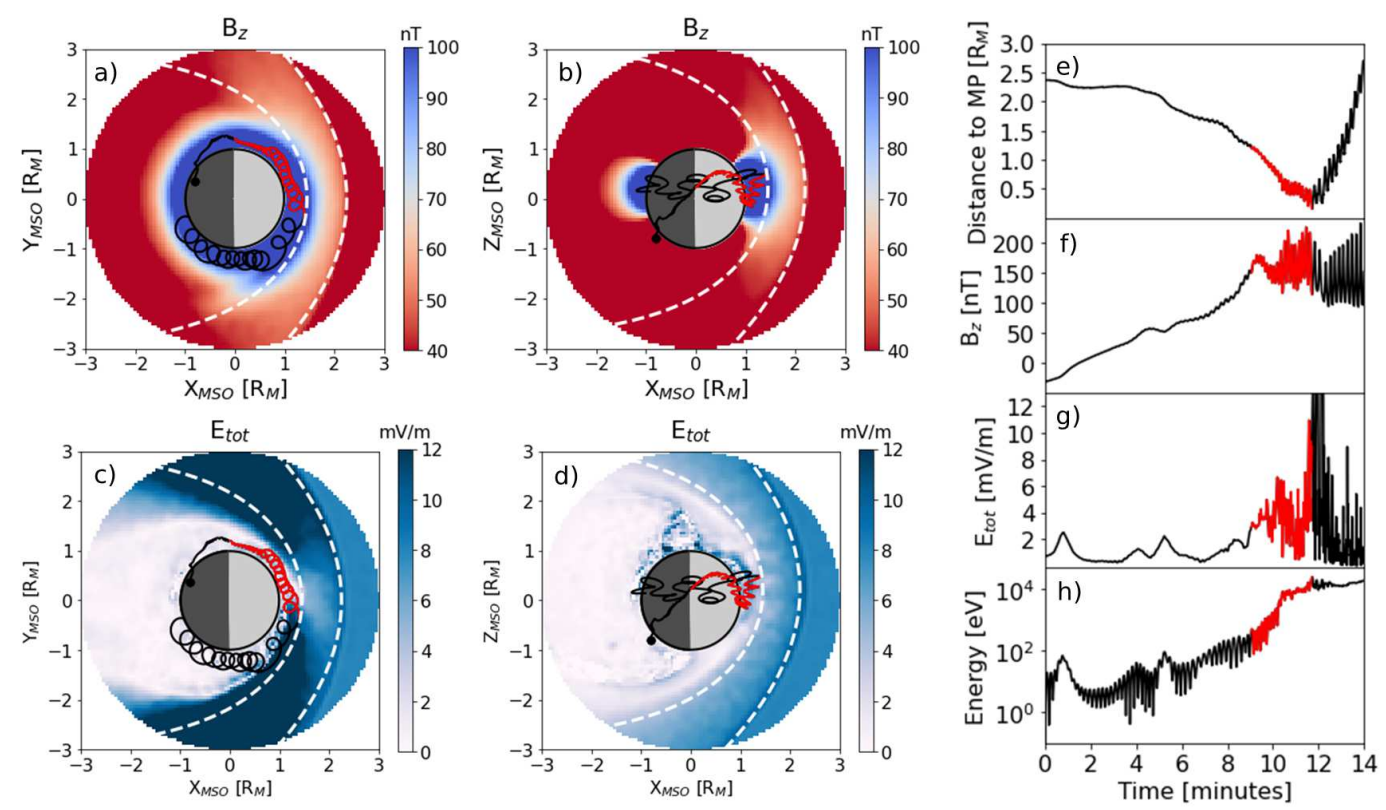

Figure 3. Example test-particle trajectory of a $\mathrm{Mg}^{+}$ion from the LIZE model in the $(\mathrm{a}, \mathrm{c})$ MSO XY-plane and the (b,d) XZ-plane. Also shown is the magnetic field component $B_{z}(\mathrm{a}, \mathrm{b})$ and the total electric field $E_{t o t}(\mathrm{c}, \mathrm{d})$ from the LathyS simulation. (e) shows the distance of the $\mathrm{Mg}^{+}$ion from the planet, (f) the time-evolution of $B_{z}$ experienced by the $\mathrm{Mg}^{+}$ion, (f) the total electric field and (h) the ion energy. The part of the test-particle trajectory highlighted in red indicates a short time period when the ion energy increases from $\sim 100 \mathrm{eV}$ to $>10 \mathrm{keV}$. The white dashed curves in (a-d) show the approximate location of the magnetopause and bow shock calculated from Winslow et al. (2013).

A and Region C. The ions in Region C are mainly sourced by magnetospheric convection from the dayside and the quasi-trapped ion drift belt.

The two peaks in the solar irradiance from the 6 September flare are relatively pronounced compared to the X8.2-class flare on the 10 September. This is caused by the difference in the optical thickness of the flare emission during the impulsive and gradual phase. The 6 September flare occurred when the active region was located near the center of the solar disk (S09W34) as seen from Earth, while the 10 September flare occurred when the active region was located near the solar limb (S08W88). The emission during the gradual phase of the flare is optically thick and more easily absorbed by the Sun's atmosphere than the impulsive emission which is optically thin. Because the optical path between an observer and the apparent solar limb is longer compared to the center of the solar disk, the intensity of the gradual flare phase emission may change considerably depending on the location of the flare source region. This means that for species like $\mathrm{He}^{+}$and $\mathrm{O}^{+}$the time of the peak photoionization frequency will also change. The $\mathrm{Mg}^{+}$photoionization frequency is mainly controlled by the impulsive flare phase and is therefore less sensitive to the location of the flare source region. It should be noted that the FISM2 flare model is based on GOES observations made at Earth, and will not reflect the true flare radiation profile at Mercury if the planet is located far away from the Sun-Earth line. The 6 September 2017 flare, for instance, erupted closer to the apparent center of the solar disk as seen from Mercury and may have caused the gradual phase flare emission to be even stronger than suggested here. 


\section{Conclusions}

We have used a test-particle model of the planetary ion density distribution in Mercury's magnetosphere which accepts time-dependent input conditions. We use this timedependent capability to model the impact of a real flare event (the X9.3-class flare on 6 September 2017) on different planetary ion species. We find the following:

- The photoionization frequency of Na was not significantly affected, while the photoionization frequencies of $\mathrm{Mg}, \mathrm{O}$ and He were increased with up to $40-80 \%$.

- The maximum He and $\mathrm{O}$ photoionization frequencies are delayed by $\sim 4$ minutes after the maximum Mg photoionization frequency. This is because the photoionization process for these species are mostly affected by the emission released during the gradual flare phase. Consequently, the photoionization frequency of $\mathrm{Mg}$ displays a relatively quick decay after the main peak compared to $\mathrm{O}$ and He.

- In the dayside magnetosphere, the low-energy ion population experiences a quicker evolution than the high-energy ions. At low altitudes on the dayside, $\sim 20 \mathrm{keV}$ energy ions take up to 14 minutes to show a flare enhancement. This comes to show that the planetary ion population experiences different dynamical evolution which have different characteristic timescales.

- In the nightside plasma sheet, there is no local ion production and ions can only be transported here from elsewhere in the magnetosphere. For this reason there is no low-energy ion population in this region. There is a time delay between the maximum ion density on the dayside and the maximum ion density in the nightside of $\sim 7$ - 8 minutes for all species.

This study shows that predicting the response of Mercury's magnetosphere to a strong solar flare is an intricate problem. What a mass spectrum analyzer on a spacecraft inside Mercury's magnetosphere will measure depends on a number of factors: the species, the location of the flare on the solar disk, the location of the spacecraft and the energy range of the instrument.

\section{Acknowledgments}

A.L.E.W., F.L., J.-Y.C. and R.M acknowledges the support by ANR of the TEMPETE project (grant ANR-17-CE31-0016). S.A., F.L., J.-Y.C., R.M and A.L.E.W. would like to acknowledge the support of CNES for the BepiColombo mission. The FISM2 model output for 6 September 2017 was obtained from the LASP Interactive Solar Irradiance Datacenter (https://lasp.colorado.edu/lisird/data/fism_flare_hr/). The LatHyS, EGM and LIZE simulation simulation results are publicly available through the IMPEx web-interface (http://impex.latmos.ipsl.fr/). To view the EGM and LIZE files, first select a LatHyS file (e.g. Data tree>Mercury $>$ Simulations > LatHyS_Merc_02_05_20), then any LatHyS, EGM or LIZE file. The files can also be downloaded directly from: http:// impex.latmos .ipsl.fr/Hybrid/Merc_02_05_20/Magw_02_05_20_t00600.nc (LatHyS magnetic field), http://impex.latmos.ipsl.fr/Hybrid/Merc_02_05_20/Elew_02_05_20_t00600 .nc (LatHyS electric field), http://impex.latmos.ipsl.fr/EGM/Mercury_05_10_21A/ EGM_Mercury_170_190_00000278_10072021.nc (EGM He), http://impex.latmos.ipsl .fr/EGM/Mercury_05_10_21B/EGM_Mercury_049_049_00800000_10072021.nc (EGM O), http://impex.latmos.ipsl.fr/EGM/Mercury_05_10_21C/EGM_Mercury_180_180_23609999 _10072021.nc (EGM Mg), http://impex.latmos.ipsl.fr/LIZE/Merc_02_05_20A/LIZE _tdep_flare_He_LatHyS_020520_EGM_170_190_00000278_t00.nc (LIZE He ${ }^{+}$), http:// impex.latmos.ipsl.fr/LIZE/Merc_02_05_20B/LIZE_tdep_flare_O_LatHyS_020520_EGM _049_049_00800000_t00.nc (LIZE O $\left.{ }^{+}\right)$and http://impex.latmos.ipsl.fr/LIZE/Merc _02_05_20C/LIZE_tdep_flare_Mg_LatHyS_020520_EGM_180_180_23609999_t00.nc (LIZE $\mathrm{Mg}^{+}$). A.L.E.W., F.L., J-Y.C. and R.M thanks the IPSL data center CICLAD for providing access to their computing resources. 


\section{References}

Aizawa, S., Griton, L. S., Fatemi, S., Exner, W., Deca, J., Pantellini, F., .. Usui, H. (2021). Cross-comparison of global simulation models applied to Mercury's dayside magnetosphere. $\quad$ Planetary and Space Science, $198 . \quad$ doi: 10.1016/j.pss.2021.105176

Anderson, B. J., Johnson, C. L., Korth, H., Purucker, M. E., Winslow, R. M., Slavin, J. A., ... Zurbuchen, T. H. (2011). The Global Magnetic Field of Mercury from MESSENGER Orbital Observations. Science, 333(6051), 1859. doi: $10.1126 /$ science.1211001

Andrews, G. B., Zurbuchen, T. H., Mauk, B. H., Malcom, H., Fisk, L. A., Gloeckler, G., .. Raines, J. M. (2007). The Energetic Particle and Plasma Spectrometer Instrument on the MESSENGER Spacecraft. Space Science Reviews, 131 (1-4), 523-556. doi: $10.1007 / \mathrm{s} 11214-007-9272-5$

Aulanier, G., DeLuca, E. E., Antiochos, S. K., McMullen, R. A., \& Golub, L. (2000). The Topology and Evolution of the Bastille Day Flare. The Astrophysical Journal, 540(2), 1126-1142. doi: 10.1086/309376

Berdermann, J., Kriegel, M., Banyś, D., Heymann, F., Hoque, M. M., Wilken, V., ... Jakowski, N. (2018). Ionospheric Response to the X9.3 Flare on 6 September 2017 and Its Implication for Navigation Services Over Europe. Space Weather, 16(10), 1604-1615. doi: 10.1029/2018SW001933

Bhardwaj, A., Elsner, R. F., Waite, J., J. Hunter, Gladstone, G. R., Cravens, T. E., \& Ford, P. G. (2005). Chandra Observation of an X-Ray Flare at Saturn: Evidence of Direct Solar Control on Saturn's Disk X-Ray Emissions. The Astrophysical Journal, 624(2), L121-L124. doi: 10.1086/430521

Bida, T. A., \& Killen, R. M. (2017). Observations of the minor species Al and Fe in Mercury's exosphere. Icarus, 289, 227-238. doi: 10.1016/j.icarus.2016.10.019

Bida, T. A., Killen, R. M., \& Morgan, T. H. (2000). Discovery of calcium in Mercury's atmosphere. Nature, 404(6774), 159-161. doi: 10.1038/35004521

Broadfoot, A. L., Kumar, S., Belton, M. J. S., \& McElroy, M. B. (1974). Mercury's Atmosphere from Mariner 10: Preliminary Results. Science, 185(4146), 166169. doi: $10.1126 /$ science.185.4146.166

Burger, M. H., Killen, R. M., McClintock, W. E., Merkel, A. W., Vervack, R. J., Cassidy, T. A., \& Sarantos, M. (2014). Seasonal variations in Mercury's dayside calcium exosphere. Icarus, 238, 51-58. doi: 10.1016/j.icarus.2014.04.049

Cassidy, T. A., McClintock, W. E., Killen, R. M., Sarantos, M., Merkel, A. W., Vervack, R. J., \& Burger, M. H. (2016). A cold-pole enhancement in Mercury's sodium exosphere. Geophysical Research Letters, 43(21), 11,121-11,128. doi: 10.1002/2016GL071071

Cassidy, T. A., Merkel, A. W., Burger, M. H., Sarantos, M., Killen, R. M., McClintock, W. E., \& Vervack, R. J. (2015). Mercury's seasonal sodium exosphere: MESSENGER orbital observations. Icarus, 248, 547-559. doi: 10.1016/j.icarus.2014.10.037

Chamberlin, P. C., Eparvier, F. G., Knoer, V., Leise, H., Pankratz, A., Snow, M., ... Woods, T. N. (2020). The Flare Irradiance Spectral Model-Version 2 (FISM2). Space Weather, 18(12). doi: 10.1029/2020SW002588

Chamberlin, P. C., Woods, T. N., Didkovsky, L., Eparvier, F. G., Jones, A. R., Machol, J. L., ... Woodraska, D. L. (2018). Solar Ultraviolet Irradiance Observations of the Solar Flares During the Intense September 2017 Storm Period. Space Weather, 16(10), 1470-1487. doi: 10.1029/2018SW001866

Chaufray, J.-. Y., Leblanc, F., Werner, A. L. E., Modolo, R., \& Aizawa, S. (2021a). Seasonal variations of $\mathrm{Mg}$ and $\mathrm{Ca}$ in the exosphere of Mercury. In Agu fall meeting abstracts 2021.

Chaufray, J.-. Y., Leblanc, F., Werner, E., Modolo, R., \& Aizawa, S. (2021b). Seasonal variations of $\mathrm{Mg}$ and $\mathrm{Ca}$ in the exosphere of Mercury. Icarus, Under review. 
Dennis, B. R., \& Schwartz, R. A. (1989). Solar Flares - the Impulsive Phase. Solar Physics, 121(1-2), 75-94. doi: 10.1007/BF00161688

Diffenbaugh, N. (Ed.). (2018). Impact of the Sept. 10, 2017, solar event on Mars [Special issue]. Geophysical Research Letters. https: //agupubs .onlinelibrary. wiley.com/doi/toc/10 .1002/(ISSN) 1944-8007.MARS SOLAR1.

Elrod, M. K., Curry, S. M., Thiemann, E. M. B., \& Jain, S. K. (2018). September 2017 Solar Flare Event: Rapid Heating of the Martian Neutral Upper Atmosphere From the X-Class Flare as Observed by MAVEN. Geophysical Research Letters, 45(17), 8803-8810. doi: 10.1029/2018GL077729

Evans, L. G., Peplowski, P. N., McCubbin, F. M., McCoy, T. J., Nittler, L. R., Zolotov, M. Y., ... Solomon, S. C. (2015). Chlorine on the surface of Mercury: MESSENGER gamma-ray measurements and implications for the planet's formation and evolution. Icarus, 257, 417-427. doi: 10.1016/j.icarus.2015.04.039

Evans, L. G., Peplowski, P. N., Rhodes, E. A., Lawrence, D. J., McCoy, T. J., Nittler, L. R., ... Goldsten, J. O. (2012). Major-element abundances on the surface of Mercury: Results from the MESSENGER Gamma-Ray Spectrometer. Journal of Geophysical Research (Planets), 117. doi: 10.1029/2012JE004178

Fallows, K., Withers, P., \& Gonzalez, G. (2015). Response of the Mars ionosphere to solar flares: Analysis of MGS radio occultation data. Journal of Geophysical Research (Space Physics), 120(11), 9805-9825. doi: 10.1002/2015JA021108

Glass, A. N., Raines, J. M., Jia, X., Tensihev, V., Shou, Y., Aizawa, S., \& A., S. J. (2021). A 3D MHD-Particle Tracing Model of Na+ Energization on Mercury's Dayside. Planetary and Space Science, 126. doi: 10.1029/2021JA029587

Jain, S. K., Deighan, J., Schneider, N. M., Stewart, A. I. F., Evans, J. S., Thiemann, E. M. B., .. Jakosky, B. M. (2018). Martian Thermospheric Response to an X8.2 Solar Flare on 10 September 2017 as Seen by MAVEN/IUVS. Geophysical Research Letters, 45(15), 7312-7319. doi: 10.1029/2018GL077731

Jasinski, J. M., Cassidy, T. A., Raines, J. M., Milillo, A., Regoli, L. H., Dewey, R., ... Murphy, N. (2021). Photoionization Loss of Mercury's Sodium Exosphere: Seasonal Observations by MESSENGER and the THEMIS Telescope. Geophysical Research Letters, 48(8). doi: 10.1029/2021GL092980

Jasinski, J. M., Regoli, L. H., Cassidy, T. A., Dewey, R. M., Raines, J. M., Slavin, J. A., ... Murphy, N. (2020). A transient enhancement of Mercury's exosphere at extremely high altitudes inferred from pickup ions. Nature Communications, 11. doi: $10.1038 / \mathrm{s} 41467-020-18220-2$

Killen, R., Cremonese, G., Lammer, H., Orsini, S., Potter, A. E., Sprague, A. L., ... Mura, A. (2007). Processes that Promote and Deplete the Exosphere of Mercury. $\quad$ Space Science Reviews, 132(2-4), 433-509. doi: 10.1007/s11214-007-9232-0

Killen, R., Shemansky, D., \& Mouawad, N. (2009). Expected Emission from Mercury's Exospheric Species, and their Ultraviolet-Visible Signatures. The Astrophysical Journal Supplement, 181(2), 351-359. doi: 10.1088/0067-0049/181/2/ 351

Knipp, D. (Ed.). $\quad$ (2018). Space Weather Events of 4-10 September 2017 [Special issue]. Space Weather. https: //agupubs .onlinelibrary.wiley.com/doi/ toc/10.1002/ ( ISSN) 1542-7390.SW-SEPT2017.

Leblanc, F., \& Chaufray, J. Y. (2011). Mercury and Moon He exospheres: Analysis and modeling. Icarus, 216(2), 551-559. doi: 10.1016/j.icarus.2011.09.028

Leblanc, F., Doressoundiram, A., Schneider, N., Mangano, V., López Ariste, A., Lemen, C., ... Cremonese, G. (2008). High latitude peaks in Mercury's sodium exosphere: Spectral signature using THEMIS solar telescope. Geophysical Research Letters, 35(18). doi: 10.1029/2008GL035322

Leblanc, F., Doressoundiram, A., Schneider, N., Massetti, S., Wedlund, M., López Ariste, A., ... Cremonese, G. (2009). Short-term variations of Mercury's Na 
exosphere observed with very high spectral resolution.

Geophysical Research Letters, 36(7). doi: 10.1029/2009GL038089

Leblanc, F., \& Johnson, R. E. (2003). Mercury's sodium exosphere. Icarus, 164(2), 261-281. doi: 10.1016/S0019-1035(03)00147-7

Leblanc, F., \& Johnson, R. E. (2010). Mercury exosphere I. Global circulation model of its sodium component. Icarus, 209(2), 280-300. doi: 10.1016/j.icarus.2010 .04 .020

Leblanc, F., Oza, A. V., Leclercq, L., Schmidt, C., Cassidy, T., Modolo, R., ... Johnson, R. E. (2017). On the orbital variability of Ganymede's atmosphere. Icarus, 293, 185-198. doi: 10.1016/j.icarus.2017.04.025

Mangano, V., Leblanc, F., Barbieri, C., Massetti, S., Milillo, A., Cremonese, G., \& Grava, C. (2009). Detection of a southern peak in Mercury's sodium exosphere with the TNG in 2005. Icarus, 201(2), 424-431. doi: 10.1016/j.icarus.2009.01.016

Mangano, V., Massetti, S., Milillo, A., Mura, A., Orsini, S., \& Leblanc, F. (2013). Dynamical evolution of sodium anisotropies in the exosphere of Mercury. Planetary and Space Science, 82, 1-10. doi: 10.1016/j.pss.2013.03.002

Mangano, V., Massetti, S., Milillo, A., Plainaki, C., Orsini, S., Rispoli, R., \& Leblanc, F. (2015). THEMIS Na exosphere observations of Mercury and their correlation with in-situ magnetic field measurements by MESSENGER. Planetary and Space Science, 115, 102-109. doi: 10.1016/j.pss.2015.04.001

Massetti, S., Mangano, V., Milillo, A., Mura, A., Orsini, S., \& Plainaki, C. (2017). Short-term observations of double-peaked Na emission from Mercury's exosphere. Geophysical Research Letters, 44(7), 2970-2977. doi: 10.1002/2017GL073090

Maurellis, A. N., Cravens, T. E., Gladstone, G. R., Waite, J. H., \& Acton, L. W. (2000). Jovian X-ray emission from solar X-ray scattering. Geophysical Research Letters, 27(9), 1339-1342. doi: 10.1029/1999GL010723

McClintock, W. E., Bradley, E. T., Vervack, R. J., Killen, R. M., Sprague, A. L., Izenberg, N. R., \& Solomon, S. C. (2008). Mercury's Exosphere: Observations During MESSENGER's First Mercury Flyby. Science, 321(5885), $92 . \quad$ doi: 10.1126/science.1159467

Mendillo, M., Withers, P., Hinson, D., Rishbeth, H., \& Reinisch, B. (2006). Effects of Solar Flares on the Ionosphere of Mars. Science, $311(5764), 1135-1138$. doi: 10.1126/science.1122099

Merkel, A. W., Cassidy, T. A., Vervack, R. J., McClintock, W. E., Sarantos, M., Burger, M. H., \& Killen, R. M. (2017). Seasonal variations of Mercury's magnesium dayside exosphere from MESSENGER observations. Icarus, 281, 46-54. doi: 10.1016/j.icarus.2016.08.032

Merkel, A. W., Vervack, R. J., Killen, R. M., Cassidy, T. A., McClintock, W. E., Nittler, L. R., \& Burger, M. H. (2018). Evidence Connecting Mercury's Magnesium Exosphere to Its Magnesium-Rich Surface Terrane. Geophysical Research Letters, 45(14), 6790-6797. doi: 10.1029/2018GL078407

Milillo, A., Mangano, V., Massetti, S., Mura, A., Plainaki, C., Alberti, T., ... Vertolli, N. (2021). Exospheric Na distributions along the Mercury orbit with the THEMIS telescope. Icarus, 355. doi: 10.1016/j.icarus.2020.114179

Modolo, R., Hess, S., Génot, V., Leclercq, L., Leblanc, F., Chaufray, J. Y., ... Holmström, M. (2018). The LatHyS database for planetary plasma environment investigations: Overview and a case study of data/model comparisons. Planetary and Space Science, 150, 13-21. doi: 10.1016/j.pss.2017.02.015

Modolo, R., Hess, S., Mancini, M., Leblanc, F., Chaufray, J.-Y., Brain, D., ... Mazelle, C. (2016). Mars-solar wind interaction: LatHyS, an improved parallel 3-D multispecies hybrid model. Journal of Geophysical Research (Space Physics), 121(7), 6378-6399. doi: 10.1002/2015JA022324

National Oceanic and Atmospheric Administration. (2011). NOAA Space Weather 
Scales. https://www.swpc.noaa.gov/sites/default/files/images/ NOAAscales.pdf. $\quad$ (Last updated on 7 April 2011. Accessed on 6 September 2021)

Neupert, W. M. (1968). Comparison of Solar X-Ray Line Emission with Microwave Emission during Flares. Astrophysical Journal, 153, L59. doi: $10.1086 / 180220$

Nittler, L. R., Starr, R. D., Weider, S. Z., McCoy, T. J., Boynton, W. V., Ebel, D. S., ... Sprague, A. L. (2011). The Major-Element Composition of Mercury's Surface from MESSENGER X-ray Spectrometry. Science, 333(6051), 1847. doi: $10.1126 /$ science. 1211567

Orsini, S., Mangano, V., Milillo, A., Plainaki, C., Mura, A., Raines, J. M., ... Aronica, A. (2018). Mercury sodium exospheric emission as a proxy for solar perturbations transit. Scientific Reports, 8. doi: 10.1038/s41598-018-19163-x

Peplowski, P. N., Lawrence, D. J., Evans, L. G., Klima, R. L., Blewett, D. T., Goldsten, J. O., ... Weider, S. Z. (2015). Constraints on the abundance of carbon in near-surface materials on Mercury: Results from the MESSENGER Gamma-Ray Spectrometer. Planetary and Space Science, 108, 98-107. doi: 10.1016/j.pss.2015.01.008

Peplowski, P. N., Lawrence, D. J., Rhodes, E. A., Sprague, A. L., McCoy, T. J., Denevi, B. W., ... Weider, S. Z. (2012). Variations in the abundances of potassium and thorium on the surface of Mercury: Results from the MESSENGER Gamma-Ray Spectrometer. Journal of Geophysical Research (Planets), 117. doi: 10.1029/2012JE004141

Potter, A. E., \& Morgan, T. H. (1985). Discovery of Sodium in the Atmosphere of Mercury. Science, 229(4714), 651-653. doi: 10.1126/science.229.4714.651

Potter, A. E., \& Morgan, T. H. (1986). Potassium in the atmosphere of Mercury. Icarus, 67(2), 336-340. doi: 10.1016/0019-1035(86)90113-2

Priest, E. R. (1981). Solar flare magnetohydrodynamics. New York: Gordon and Breach.

Raines, J. M., Gershman, D. J., Slavin, J. A., Zurbuchen, T. H., Korth, H., Anderson, B. J., \& Solomon, S. C. (2014). Structure and dynamics of Mercury's magnetospheric cusp: MESSENGER measurements of protons and planetary ions. Journal of Geophysical Research (Space Physics), 119(8), 6587-6602. doi: 10.1002/2014JA020120

Raines, J. M., Gershman, D. J., Zurbuchen, T. H., Sarantos, M., Slavin, J. A., Gilbert, J. A., ... Solomon, S. C. (2013). Distribution and compositional variations of plasma ions in Mercury's space environment: The first three Mercury years of MESSENGER observations. Journal of Geophysical Research: Space Physics, 118(4), 1604-1619. doi: 10.1029/2012JA018073

Raines, J. M., Wallace, K. L., Sarantos, M., Jasinksi, J. M., Tracy, P. J., Dewey, R. M., ... Slavin, J. A. (2018). First In-Situ Observations of Exospheric Response to CME Impact at Mercury. In Mercury: Current and future science of the innermost planet (Vol. 2047).

Thiemann, E. M. B., Andersson, L., Lillis, R., Withers, P., Xu, S., Elrod, M., ... Deighan, J. (2018). The Mars Topside Ionosphere Response to the X8.2 Solar Flare of 10 September 2017. Geophysical Research Letters, 45(16), 8005-8013. doi: 10.1029/2018GL077730

Tsurutani, B. T., Judge, D. L., Guarnieri, F. L., Gangopadhyay, P., Jones, A. R., Nuttall, J., ... Viereck, R. (2005). The October 28, 2003 extreme EUV solar flare and resultant extreme ionospheric effects: Comparison to other Halloween events and the Bastille Day event. Geophysical Research Letters, 32(3). doi: 10.1029/2004GL021475

Verner, D. A., Ferland, G. J., Korista, K. T., \& Yakovlev, D. G. (1996). Atomic Data for Astrophysics. II. New Analytic FITS for Photoionization Cross Sections of Atoms and Ions. The Astrophysical Journal, 465, 487. doi: 
$10.1086 / 177435$

Vervack, R. J., Killen, R. M., McClintock, W. E., Merkel, A. W., Burger, M. H., Cassidy, T. A., \& Sarantos, M. (2016). New discoveries from MESSENGER and insights into Mercury's exosphere. Geophysical Research Letters, 43(22), 11,545-11,551. doi: 10.1002/2016GL071284

Werner, A. L. E., Aizawa, S., Leblanc, F., Chaufray, J.-. Y., Modolo, R., Raines, J. M., ... Schmidt, C. (2022). Ion density and phase space density distribution of planetary ions $\mathrm{Na}^{+}, \mathrm{O}^{+}$and $\mathrm{He}^{+}$in Mercury's magnetosphere. Icarus, 372 . doi: 10.1016/j.icarus.2021.114734

Winslow, R. M., Anderson, B. J., Johnson, C. L., Slavin, J. A., Korth, H., Purucker, M. E., ... Solomon, S. C. (2013). Mercury's magnetopause and bow shock from MESSENGER Magnetometer observations. Journal of Geophysical Research (Space Physics), 118(5), 2213-2227. doi: 10.1002/jgra.50237

Yagi, M., Seki, K., Matsumoto, Y., Delcourt, D. C., \& Leblanc, F. (2010). Formation of a sodium ring in Mercury's magnetosphere. Journal of Geophysical Research (Space Physics), 115(A10). doi: 10.1029/2009JA015226

Yan, X. L., Wang, J. C., Pan, G. M., Kong, D. F., Xue, Z. K., Yang, L. H., ... Feng, X. S. (2018). Successive X-class Flares and Coronal Mass Ejections Driven by Shearing Motion and Sunspot Rotation in Active Region NOAA 12673. The Astrophysical Journal, 856(1), 79. doi: 10.3847/1538-4357/ aab153

Zurbuchen, T. H., Raines, J. M., Slavin, J. A., Gershman, D. J., Gilbert, J. A., Gloeckler, G., ... Solomon, S. C. (2011). MESSENGER Observations of the Spatial Distribution of Planetary Ions Near Mercury. Science, 333(6051), 1862. doi: $10.1126 /$ science. 1211302 
Figure 1. 
Figure 3. 
Figure 2. 

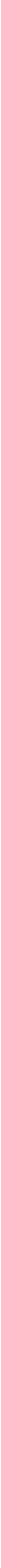